\title{
Tablet-PC: Flexible Lösungen für den Arzt
}

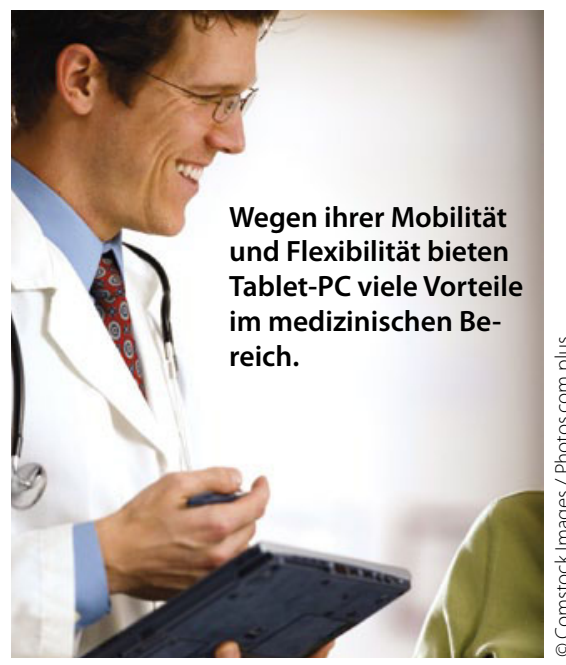

- Spätestens seit Apples iPad auf dem Markt ist, sind Tablet-PC überall Gesprächsstoff. Die kleinen Multitalente bieten gerade für Arztpraxen interessante Eigenschaften. Wer dabei nur an Hausbesuche denkt, steckt das Einsatzgebiet für die flachen, mobilen PC viel zu eng ab. Spannend ist es natürlich, auf Hausbesuch statt mit den oft schwereren Notebooks mit einem Tablet-PC vorbeizuschauen. Das iPad wiegt je nach Modell bis zu 0,73 Kilogramm, der Samsung Galaxy Tab gerade einmal 380 Gramm. Dabei lassen sich alle wichtigen Funktionen für den Besuch auf den Tablet-PC unterbringen.

Die Tablet-PC erlauben es aber vor allem, am Krankenbett, beim Hausbesuch oder in der Sprechstunde Patienten Therapien und Diagnose schnell mithilfe von Bildern, Videos oder Grafiken zu erklären. Der Arzt nimmt das Gerät einfach von Zimmer zu Zimmer mit, d. h., nicht in jedem Raum muss also ein eigener Rechner stehen.

Da viele Geräte mit Googles AndroidBetriebssystem oder gar gleich mit

\section{Auf dem Markt der Tablet-PC tut sich so einiges. Die Geräte lassen sich gerade im Praxisalltag und auf Hausbesuch gut einsetzen. Spezielle Lösungen und Module halten die Anbieter längst bereit.}

einem Windows-Betriebssystem laufen, können Office-Dokumente problemlos geöffnet und gespeichert werden. Selbst Apples iPad kann mit Windows laufen. Und die Handhabung der Tablet-PC ist meist einfach und schnell gelernt. Auch einige Praxis-EDV-Anbieter haben längst reagiert: Sie bieten mobile Versionen ihrer Programme, zum Teil spezielle Hausbesuchsmodule wie etwa MCSISYNET oder TurboMed, oder sogar die Software fürs iPad wie Frey ADV mit Quincy-Win.

\section{Spezialgerät für den Gesundheitssektor}

Speziell für den Gesundheitsbereich entwickelt wurde der Motion C5v von Motion Computing. Das Gerät verfügt über eine $160 \mathrm{~Gb}-\mathrm{HD}$ und einen Arbeitsspeicher von bis zu vier GB. Integriert ist ein RFID-Scanner (RFID = radio-frequency identification), mit dem sich Ärzte in Kliniken beispielsweise für den Zugriff via RFID-Transponder identifizieren können oder die RFIDCodes von Patienten einlesen und gleich die richtige Patientenakte aufrufen können.

Einige Tablet-PC - der C5v und auch der Partner EM-200 - können sogar mit einem SmartCard-Lesegerät erweitert werden. Das ist für das Einlesen von Versichertenkarten, Heilberufsausweisen oder das elektronische Signieren von Dokumenten interessant, und könnte spätestens zur Einführung der elektronischen Gesundheitskarte (eGK) spannend werden, sofern die Kartenleser auch eGK-fähig sind. Die Eingabe von Daten erfolgt über mehrere Wege:
Die meisten Geräte besitzen gleich ein Mikrofon und mindestens eine Kamera. So etwa der Touchlet X2, dessen Arbeitsspeicher sich per micro SD-Karte auf 32 GB erweitern lässt. Der Dell Streak besitzt gleich zwei Kameras: eine auf der Vorderseite - etwa für Videokonferenzen - und eine auf der Rückseite. Die integrierten Kameras ermöglichen die einfache Bilddokumentation von Krankheits- und Therapieverläufen.

\section{Nützliche Zusatzfunktionen}

Natürlich lassen sich Daten per Bildschirm-Tastatur über den Touchscreen eingeben. Wer allerdings lieber eine echte Tastatur nutzt, kann auch diese an die meisten Geräte anschließen. Wobei der Dell Streak ähnlich wie das Samsung Galaxy Tab eine Besonderheit hat: Beide Geräte sind eigentlich Zwitter, weil Tablet-PC und Smartphone in einem. Das heißt, Ärzte können von unterwegs mit ihnen auch telefonieren.

Das Samsung Galaxy Tab wiederum bietet die Videowiedergabe in High-Definition-Qualität, also besonders hoher Bildauflösung. Neu auf den Markt kommt bei der CeBIT das G-Slate von LG. Das Gerät kommt in der Grundausstattung mit drei Kameras daher und macht 3-D-Aufnahmen möglich.

Daten mit dem Praxisrechner lassen sich meist per Bluetooth, USB-Kabel oder gesicherter WLAN-Verbindung austauschen. Aber: An USB-Anschlüssen mangelt es vielen Geräten, hier - etwa auch beim iPad (mit dem Dock Connector-zu-USB-Kabel) - müssen Ärzte oft mit speziellen Connector-Kabeln arbeiten. 